\title{
Dosage des stupéfiants dans le sang des conducteurs impliqués dans un accident de la circulation : interprétation des résultats, définition de seuils
}

\section{Determination of narcotics in blood after traffic accidents : interpretation of the results and advice for cut-offs}

Marc DEVEAUX*(1), Jean-Pierre GOULLÉ( ${ }^{(2)}$, Michel LHERMITTE ${ }^{(3)}$

(1) Institut de Médecine Légale, Faculté de Médecine, Université de Lille II, rue André Verhaeghe - 59045 LILLE Cedex (2) Laboratoire de Pharmacologie et de Toxicologie, Groupe Hospitalier - 76083 LE HAVRE Cedex (3) Laboratoire de Biochimie, de Biologie Moléculaire et de Toxicologie, Hôpital Calmette, CHRU - 59037 LILLE Cedex

* Auteur à qui adresser la correspondance : Marc DEVEAUX, Institut de Médecine Légale, Faculté de Médecine, Université de Lille II, rue André Verhaeghe - 59045 LILLE Cedex - FRANCE Tél : 0320623510 - Fax 0320623512 - E-mail : mdeveaux@easyconnect.fr

(Reçu le 25 avril 2003 ; accepté-le 9 mai 2003)

\section{RÉSUMÉ}

De 1995 à 2001, la gestation de la législation française pour la prévention et la répression de la conduite sous l'empire de stupéfiants a été longue. Nous détaillons dans cet article les dispositions finales, applicables depuis avril 2003 : prélèvements, dépistage urinaire, dosages sanguins. Les méthodes d'analyses recommandées par la Société Française de Toxicologie Analytique publiées en 1996 sont toujours d'actualité, avec quelques améliorations dues essentiellement aux progrès du matériel et à l'expérience des toxicologues. La législation manquant de précision scientifique, nous proposons un choix précis de xénobiotiques à rechercher et à doser : tétrahydrocannabinol, 11-hydroxy-tétrahydrocannabinol, benzoylecgonine, morphine libre, 6-monoacétylmorphine, méthylène-dioxyméthamphétamine. Nous discutons les seuils imposés par la législation et nous en proposons d'autres, plus bas, fondés sur les limites analytiques de quantification les plus couramment admises par les laboratoires de référence.

MOTS-CLÉS

conduite automobile, stupéfiants, seuils.

\section{SUMMARY}

It took 6 years, from 1995 to 2001 to build up the French legislation on narcotic drugs and driving. This paper describes the actual law published in April 2003 : biological samples, urinalysis, determination of drugs in blood. Recommended analytical procedures previously published in 1996 by the French Society of Analytical Toxicology (Société Française de Toxicologie Analytique, SFTA) are still utilizable, but are improved by progress in apparatus and toxicologists' experience. The actual law appears to have not enough scientific accuracy and we propose in this paper which precise xenobiotics to determine : tetrahydrocannabinol, 11-hydroxy-tetrahydrocannabinol, benzoylecgonine, free morphine, 6-monoacetylmorphine, methylenedioxymethamphetamine. Authors discuss the cut-offs imposed in the law and propose lower cut-offs, based on analytical performances of most of the reference laboratories.

\section{KEY-WORDS}

driving under influence, narcotics, cut-offs. 


\section{Introduction}

Au printemps 1995, la commission «drogues, psychotropes et sécurité routière» de la Société Française de Toxicologie Analytique (SFTA) avait été chargée de proposer aux pouvoirs publics un milieu biologique et une méthodologie adaptés pour la recherche et le dosage des substances psychotropes licites ou illicites chez les conducteurs impliqués dans un accident de la circulation. La réponse a été apportée un an après (1), et elle est résumée ici : 4 familles de stupéfiants doivent être recherchées (cannabinoïdes, amphétamines, cocaïne et opiacés), en commençant par un dépistage dans l'urine, suivi en cas de positivité par une confirmation et une quantification obligatoires dans le sang. Pour cela des méthodes de dosage ont été recommandées sans être imposées, avec au minimum l'utilisation d'un couplage chromatographie gazeuse/spectrométrie de masse (CPG/SM) (2-4). Enfin, un contrôle externe de qualité pour le dosage dans le sang de stupéfiants faisant partie de ces 4 familles a été mis en place par la SFTA (5).

Il a fallu attendre août 2001 et la loi Gayssot, ses décrets et ses arrêtés d'application pour que ces dispositions soient reprises par le législateur $(6,7)$ qui a alors initié une «étude épidémiologique» devant se dérouler sur 2 ans. Les premiers résultats ont dû paraître suffisants puisque une dernière modification vient d'être publiée, ne rendant plus obligatoire la recherche et le dosage de médicaments psychoactifs et réservant les dosages de stupéfiants dans le sang à des experts judiciaires (8-10). Nous verrons ci-après le détail des dispositions législatives, les techniques à utiliser et proposerons une aide à l'interprétation des résultats.

\section{Recherches mises en jeu après un accident}

Pour le législateur, est considéré comme accident mortel tout accident ayant eu des conséquences immédiatement mortelles (9). La notion d'immédiateté n'est pas définie, mais dans une circulaire aux parquets, la Chancellerie a précisé qu'elle s'étend jusqu'à l'admission dans une structure médicale. Cela a le mérite de définir clairement le champ d'application, mais laisse de côté tous les accidents mortels au sens des assurances, c'est-à-dire quand le décès d'un blessé survient dans les 24 à 72 heures qui suivent. La comparaison des résultats avec ceux d'autres pays européens et la comparaison avec d'autres facteurs d'accidents mortels devront donc être très prudentes. Cependant, un article de loi passé relativement inaperçu permettait déjà d'effectuer ces recherches dans le cas d'accidents corporels (11). Depuis la loi du 3 février 2003, c'est systématique si l'on soupçonne que le conducteur a fait usage de stupéfiants, et cette loi étend même le champ d'application à tous les accidents de la circulation (8).

Les prélèvements et les recherches suivants seront donc effectués sur tous les conducteurs impliqués dans l'accident :

- sur les conducteurs vivants : examen clinique par un médecin (fiches A et B), évaluation de l'état d'imprégnation alcoolique par les méthodes habituelles (éthylotest et/ou éthylomètre et/ou prise de sang puis dosage de l'alcoolémie $(5,12)$ ), recueil d'urine et recherche des 4 familles de stupéfiants. Ce dépistage urinaire se fait dans une srtucture hospitalière et il est précédé d'un examen clinique dont les items sont différents du précédent (fiches $\mathrm{D}$ et $\mathrm{E}$ ). Si les résultats du dépistage des stupéfiants sont négatifs, les investigations s'arrêtent. Si un des résultats est positif dans l'urine, il est alors procédé à une prise de sang $(2 \times 10 \mathrm{~mL}$ sur héparinate de lithium). Sur ces prélèvements sanguins seront alors pratiqués la recherche et le dosage de stupéfiants.

- sur les conducteurs décédés : les prélèvements sanguins sont effectués directement dans les mêmes conditions, ou par un médecin légiste lors de l'autopsie judiciaire. Le prélèvement se fait alors sur fluorure de sodium. Il arrive fréquemment, surtout quand ce prélèvement n'est pas fait par un médecin légiste, que la quantité disponible soit très insuffisante pour les analyses. Il convient alors de demander par téléphone à l'officier de police judiciaire ou au procureur de la République requérant, l'autorisation d'utiliser pour les premières analyses tous les prélèvements (à charge pour l'expert de conserver si possible une fraction pour l'éventuelle contre-expertise). La confirmation écrite de cette demande est indispensable. Il est donc préférable que ce soit le même expert qui fasse le dosage d'alcool ( $2 \times 5 \mathrm{~mL}$ de sang ont été prélevés) et le dosage de stupéfiants, car il dispose ainsi d'un ensemble de prélèvements sanguins effectués au même moment.

Les recherches toxicologiques à effectuer sont précisées sur la fiche F. Les données scientifiques correspondant aux données de la fiche $\mathrm{F}$ sont indiquées entre parenthèses : $\Delta$-9-THC (cannabinoïdes), amphétamine (amphétamines, notamment la MDMA et ses dérivés), cocaïne (métabolites de la cocaïne), opiacés (héroïne, 6-monoacétylmorphine, médicaments morphiniques). Il faut obligatoirement utiliser le couplage CPG/SM (6), mais on ne pourrait reprocher à un expert d'utiliser des couplages plus performants comme la chromatographie liquide/spectrométrie de masse (CLHP/SM) et des détecteurs de masse en tandem : l'essentiel est bien de pouvoir identifier de façon absolue le ou les stupéfiants retrouvés. Depuis la parution du décret $n^{\circ} 2003-$ 293 (9), la recherche des médicaments psychoactifs ne 
doit être faite qu'à la demande du conducteur et non plus systématiquement en cas de positivité de la recherche de stupéfiants. Si elle est faite, cette recherche doit être faite par CPG/SM et chromatographie liquide/barette de diodes (CLHP/BD). Rien ne précise si elle n'est que qualitative ou s'il faut également quantifier les médicaments psychotropes mis en évidence.

Les fiches D, E, et $F$ encore en vigueur en mai 2003 sont reproduites en annexe. Les fiches A, B et C n'ont pas été modifiées depuis leur reproduction dans la référence (12).

\section{Méthodes à utiliser}

Les méthodes validées et proposées en 1996 (2-5) sont toujours d'actualité, mais chaque toxicologue a pu les améliorer en fonction de ses habitudes de travail et de son matériel. L'extraction en phase solide s'est notamment fortement développée. Nous les détaillons ci-dessous, en proposant quelques variantes.

\section{Opiacés, cocaïne et métabolites}

A $1 \mathrm{~mL}$ de sang sont ajoutés 100 à $200 \mathrm{ng}$ de chaque étalon interne trideutéré sous un volume minimal (20 à $100 \mu \mathrm{L}$ ). Le milieu est alcalinisé par addition de $1 \mathrm{~mL}$

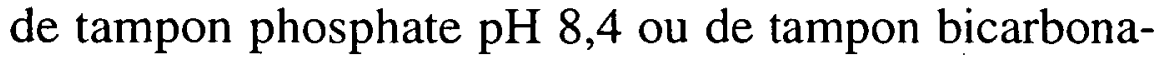
te $\mathrm{pH} 8,6$. L'extraction peut être au choix liquide/liquide ou solide/liquide.

Dans le premier cas, on utilise 8 à $10 \mathrm{~mL}$ du mélange classique chloroforme/n-heptane/isopropanol (50:33: $17, \mathrm{v} / \mathrm{v})$ ou de chloroforme/isopropanol $(9: 1, \mathrm{v} / \mathrm{v})$. La phase organique est lavée par $2 \mathrm{~mL}$ d' $\mathrm{HCl} 0,2 \mathrm{~N}$. La phase aqueuse est récupérée, neutralisée par $0,4 \mathrm{~mL}$ de soude $1 \mathrm{~N}$ et tamponnée par $1 \mathrm{~mL}$ de tampon phosphate ; elle est ensuite réextraite par $5 \mathrm{~mL}$ de chloroforme/isopropanol. Après centrifugation, le mélange est évaporé à sec, sans chauffage excessif.

Dans le deuxième cas, le sang doit être dilué avec $1 \mathrm{~mL}$ d'eau distillée avant d'être tamponné. La colonne est conditionnée par du méthanol et du tampon bicarbonate. Les analytes sont élués par du méthanol. L'éluat est évaporé à sec.

La dérivation se fait directement sur les extraits secs par $50 \mu \mathrm{L}$ de $N, O$-bis(triméthylsilyl)trifluoroacétamide (BSTFA) catalysé par $1 \%$ de triméthylchlorosilane (TMCS), à $70^{\circ} \mathrm{C}$ pendant 20 à $60 \mathrm{~min}$. Un à $2 \mu \mathrm{L}$ sont injectés dans la colonne analytique. On utilisera de préférence une colonne capillaire greffée apolaire $5 \%$ phényl-95\% méthyl siloxane de 25 à $30 \mathrm{~m}$ de long et d'épaisseur de film $0,25 \mu \mathrm{m}$, à faible bruit de fond. Les ions qualifiants et les ions quantifiants sont donnés dans le tableau I.

\section{Amphétamines}

L'analyse est réalisée à partir de $1 \mathrm{~mL}$ de sang auquel on ajoute $100 \mathrm{ng}$. de chaque étalon interne deutéré. L'extraction se fait par $5 \mathrm{~mL}$ de diéthyl éther ou d'acétate d'éthyle après alcalinisation par $0,5 \mathrm{~mL}$ de soude $1 \mathrm{~N}$. Après agitation et centrifugation, la phase organique est récupérée, additionnée de $100 \mu \mathrm{L}$ du mélange méthanol/HCl $(99: 1)$ puis évaporée sous azote. Deux méthodes de dérivation peuvent s'appliquer : ajout de 50 à $100 \mu \mathrm{L}$ d'anhydride heptafluorobutyrique (HFBA) dans $50 \mu \mathrm{L}$ d'acétate d'éthyle, avec incubation à 60$70^{\circ} \mathrm{C}$ pendant 20 à $30 \mathrm{~min}$, ou par $100 \mu \mathrm{L}$ d'anhydride trifluoroacétique (TFAA) dans $100 \mu \mathrm{L}$ d'acétonitrile avec incubation $30 \mathrm{~min}$ à $60^{\circ} \mathrm{C}$. Une étape facultative de lavage peut être faite à ce stade : la phase organique est évaporée sous azote, reprise par $400 \mu \mathrm{L}$ d'hexane et lavée par de l'eau distillée (200 $\mu \mathrm{L})$ puis de l'ammoniaque à $4 \%(200 \mu \mathrm{L})$. La phase organique est enfin évaporée à sec et reprise par $50 \mu \mathrm{L}$ d'acétate d'éthyle. On injecte $1 \mu \mathrm{L}$ dans la colonne analytique qui est la même que celle utilisée pour les opiacés. Les ions qualifiants et les ions quantifiants sont donnés dans le tableau I.

\section{Cannabinoïdes}

Pour ces dosages, toute la verrerie doit impérativement être silanisée. Il est plus confortable de l'acheter prête à l'emploi. A $1 \mathrm{~mL}$ de sang on ajoute $20 \mathrm{ng}$ de chaque étalon interne trideutéré. On peut ou non ajouter $1 \mathrm{~mL}$ d'eau distillée. L'échantillon est acidifié par $200 \mu \mathrm{L}$ d'acide acétique à $10 \%$. L'extraction est effectuée par $5 \mathrm{~mL}$ du mélange hexane/acétate d'éthyle $(9: 1, \mathrm{v} / \mathrm{v})$. L'addition de $200 \mu \mathrm{L}$ d'alcool isoamylique est facultative à ce stade. Après obtention d'un résidu sec, on peut utiliser l'une ou l'autre des méthodes de dérivation suivantes : l'une classique par $50 \mu \mathrm{L}$ de BSTFA, l'autre étant une méthylation, plus longue, par addition de 200 $\mu \mathrm{L}$ d'hydroxyde de tétraméthylammonium-diméthylsulfoxyde (TMAH-DMSO) et de $50 \mu \mathrm{L}$ d'iodure de méthane, arrêt par $200 \mu \mathrm{L}$ d'HCl $0,1 \mathrm{~N}$, extraction par $1 \mathrm{~mL}$ d'isooctane, évaporation, reprise par $25 \mu \mathrm{L}$ d'isooctane. On injecte $2 \mu \mathrm{L}$ dans le système chromatographique. Les ions qualifiants et les ions quantifiants sont donnés dans le tableau I.

\section{Interprétation des résultats, seuils}

\section{Problématique}

Pour le dépistage, l'article 4 de l'arrêté du 5 septembre 2001 (7) fixe des seuils minima de détection des stupéfiants dans les urines : $\Delta-9-\mathrm{THC} 50 \mathrm{ng} / \mathrm{mL}$, amphéta- 
Tableau I : Ions de qualification et de quantification pour les molécules à doser en CPG/SM.

\begin{tabular}{|c|c|c|}
\hline \multirow[t]{2}{*}{ Molécule } & \multicolumn{2}{|c|}{ ions $(m / z)$ après dérivation ions $(m / z)$ avec d'autres } \\
\hline & selon les réf. (2-5) & modes de dérivation \\
\hline Benzoylecgonine & $82, \underline{240}, 361$ & \\
\hline Ecgonine méthyl ester & $82,96,271$ & \\
\hline Cocaéthylène & $82,196,317$ & \\
\hline Cocainne & $82, \underline{182}, 303$ & \\
\hline Codéine & $234, \underline{371}$ & \\
\hline Morphine & $236,414, \underline{429}$ & \\
\hline 6-acétylmorphine & $340, \underline{399}$ & \\
\hline Codéthyline & $234, \underline{385}$ & \\
\hline Pholcodine & $100, \underline{114}$ & \\
\hline Dihydrocodéine & $178,236, \underline{373}$ & \\
\hline Amphétamine & $91,118, \underline{240}$ & $91,118, \underline{140}$ \\
\hline Méthamphétamine & $91,169,210, \underline{254}$ & $110,118, \underline{154}$ \\
\hline MDMA & $162,210, \underline{254}, 389$ & $135, \underline{154}, \overline{162}$ \\
\hline MDA & $135,162,240, \underline{375}$ & $\underline{135}, \overline{162}$ \\
\hline MDEA & $240, \underline{268}, 403$ & $140,162,1 \underline{68}$ \\
\hline MBDB & $176, \underline{268}, 403$ & $135,168, \underline{176}$ \\
\hline Ephedrine & $210, \underline{254}$ & $110, \underline{154}$ \\
\hline$\Delta-9-\mathrm{THC}$ & $285,313, \underline{328}$ & $315,371, \underline{386}$ \\
\hline$\Delta-9-\mathrm{THC}-\mathrm{COOH}$ & $\underline{313}, 357,372$ & $371, \underline{473}, 488$ \\
\hline $11-\mathrm{OH}-\Delta-9-\mathrm{THC}$ & $313,314, \underline{358}$ & $\underline{371}, 459,474$ \\
\hline
\end{tabular}

Les ions de quantification sont soulignés. Les ions des étalons internes deutérés utilisés pour la quantification ont une masse supérieure de 3,5 ou 9 selon le cas. Les ions de quantification sont différents selon le mode de dérivation pour les amphétamines (HFBA ou TFAA) et les cannabinoïdes (BSTFA ou TMAH). Les abréviations sont données dans le texte.

mines $1000 \mathrm{ng} / \mathrm{mL}$, cocaïne $300 \mathrm{ng} / \mathrm{mL}$, opiacés $300 \mathrm{ng} / \mathrm{mL}$. Ce sont des seuils de positivité qui sont ainsi définis, et les tests disponibles sur le marché permettent souvent de descendre en dessous. Cependant la sensibilité de chaque test immunochimique dépend de la ou des molécules présentes dans les urines.

Le problème est plus complexe pour le dosage des stupéfiants dans le sang. Les articles 10 et 11 du même arrêté (7) précisent que la recherche et le dosage des stupéfiants dans le sang doit se faire obligatoirement par CPG/SM en respectant les seuils minima de détection suivants : $\Delta$-9-THC $1 \mathrm{ng} / \mathrm{mL}$, amphétamines $50 \mathrm{ng} / \mathrm{mL}$, cocaïne $50 \mathrm{ng} / \mathrm{mL}$, opiacés $20 \mathrm{ng} / \mathrm{mL}$. Conformément aux propositions faites en 1996 par la SFTA (1), ce sont bien des seuils analytiques minima qui ont été fixés par l'arrêté. S'agissant de produits stupéfiants illicites, la notion de concentration sanguine légalement tolérable n'avait bien entendu pas de sens. Il est, à l'heure actuelle, quasiment impossible de définir précisément des seuils pharmacologiques, donnant pour chaque molécule la concentration sanguine minimale ayant un effet sur les capacités à conduire (13). Grâce aux efforts des toxicologues analystes et aux industriels, on a montré que les seuils analytiques sont entièrement dépendants des appareils, des méthodes et des laboratoires. Nous verrons pour chaque famille de stupéfiants ce qu'il en est actuellement.

\section{Cannabinoïdes}

On retrouve dans le sang des composés actifs $(\Delta-9$ THC et 11-hydroxy- $\Delta-9-$ THC) et un composé inactif, le $\Delta-9$-THC-COOH. On peut détecter le $\Delta-9$-THC pendant 3 à 12 heures, le pic sanguin étant atteint dans les 10 min suivant l'inhalation. Le 11-hydroxy- $\Delta-9$-THC est détectable pendant seulement 4 à 5 heures, avec des concentrations 10 fois plus faibles. En revanche le $\Delta-9$ THC-COOH est détectable pendant environ 48 à 168 heures $(14,15)$. On sait maintenant qu'il y a un décalage dans le temps entre l'évolution de la concentration sanguine du $\Delta-9-\mathrm{THC}$ et les effets physiques et psychiques ressentis par le consommateur : ces effets sont maximum environ $1 \mathrm{~h}$ après le pic sanguin de $\Delta-9$-THC et ils se prolongent pendant environ $2 \mathrm{~h}$, alors que la concentration sanguine de $\Delta-9$-THC est de l'ordre du $\mathrm{ng} / \mathrm{mL}(15,16)$. Il n'y a donc pas de relation forte et bien définie entre les concentrations sanguines de $\Delta-9$ THC et de 11-OH- $\Delta-9-$ THC et les effets.

Il existe deux modèles mathématiques proposés pour estimer le délai écoulé entre la dernière consommation et le prélèvement, et tenter de corréler les effets aux concentrations (revue générale dans (15)) : leur utilisation nécessite le dosage dans le sang des trois composés $\Delta-9$-THC, $11-\mathrm{OH}-\Delta-9-\mathrm{THC}$ et $\Delta-9$-THC-COOH. De plus une concentration en métabolite hydroxylé nettement supérieure à celle du $\Delta-9$-THC permet d'évoquer une consommation par voie orale (17). Il est donc primordiàl de pouvoir identifier et doser les 3 composés précités. L'expérience a montré que $95 \%$ des sujets avaient une concentration sanguine en $\Delta-9$-THC supérieure à $0,5 \mathrm{ng} / \mathrm{mL}(18,19)$ : il faut donc pouvoir doser des concentrations de $\Delta-9-\mathrm{THC}$ d' au moins $0,5 \mathrm{ng} / \mathrm{mL}$. La limite de quantification du $\Delta-9$-THC dans le sang est actuellement de 0,2 $\mathrm{ng} / \mathrm{mL}$ (4) (tableau II).

\section{Amphétamines}

Cette famille chimique rassemble au moins une vingtaine de produits différents et elle est en constante évolution $(20,21)$. Les plus fréquemment utilisés sont la 3,4-méthylènedioxyméthamphétamine (MDMA, ecstasy) et la 3,4-méthylènedioxyéthamphétamine (MDEA). La 3,4-méthylène dioxyamphétamine (MDA) et la méthylbenzodioxazolylbutanamine (MBDB) sont d'un usage plus restreint. La MDA est un métabolite commun à la MDEA et à la MDMA. Les données de pharmacocinétiques ne sont bien établies que pour la MDMA $(21,22)$. On estime que le pic sanguin est atteint entre 2 et $4 \mathrm{~h}$ après une prise orale de $50 \mathrm{mg}$ de MDMA (51 à $106 \mathrm{ng} / \mathrm{mL}$ ). Pour une dose de $100 \mathrm{mg}$ il est compris entre 190 et $331 \mathrm{ng} / \mathrm{mL}$. La demi-vie san- 
guine de ces composés oscille entre 4 et $12 \mathrm{~h}$ et elle est dose-dépendante. Huit heures après une prise orale de $100 \mathrm{mg}$, on peut encore détecter l'ecstasy dans le sang. Les effets physiques et psychiques sont fortement liés aux concentrations sanguines, selon un modèle tout à fait comparable à celui de l'alcool éthylique.

Les limites de quantification que l'ont peut atteindre sont actuellement les suivantes : MDMA et MDEA 5 $\mathrm{ng} / \mathrm{mL}$, MDA $20 \mathrm{ng} / \mathrm{mL}$, amphétamine $10 \mathrm{ng} / \mathrm{mL}$ (3, 21). Comme les limites de détection et de quantification ne sont pas identiques pour toutes les molécules de cette vaste famille chimique, il est difficile de fixer un seuil analytique unique : il devrait être discuté ultérieurement molécule par molécule (tableau II).

\section{Opiacés}

La famille des opiacés présente la particularité d'avoir des représentants licites et des représentants illicites. Les médicaments licites sont d'un usage courant : les antitussifs peuvent être obtenus sans ordonnance (codéine, codéthyline, pholcodine), les antalgiques majeurs quant à eux ne peuvent obtenus que sur ordonnance sécurisée (morphiniques du palier III : morphine, hydromorphone, oxycodone, dihydrocodéine). Les relations doses-effets de tous ces produits utilisés en thérapeutique depuis longtemps sont bien établies (22, 23). Les effets positifs sont essentiellement un apaisement des douleurs intenses; les effets négatifs pour la conduite automobile sont la somnolence et des secousses musculaires involontaires (23). Cependant les pathologies traitées par les morphiniques forts sont la plupart du temps incompatibles avec la conduite automobile. Les effets antalgiques de la morphine sont bien identifiés à partir d'une concentration sanguine de $10 \mathrm{ng} / \mathrm{mL}$ de morphine libre, mais la fourchette thérapeutique va de 10 à $70 \mathrm{ng} / \mathrm{mL}$. La demi-vie de la mor- phine est de 2 à 3 heures environ. L'hérö̈ne est le seul opiacé semi-synthétique illicite et les doses administrées sont éminemment variables. Son métabolite intermédiaire et caractéristique est la 6-monoacétylmorphine, dont la demi-vie est d'une trentaine de minutes. Toutes ces molécules opiacées ont des métabolites donnant une réponse positive avec les tests immunochimiques de détection urinaire. La recherche et le dosage dans le sang de la molécule mère et/ou des métabolites libres et conjugués sont donc impératives. Le problème des métabolites est complexe car certains individus (environ $7 \%$ de la population) ne métabolisent pas la codéine en morphine (24) et on peut observer des concentrations sanguines de pholcodine ou de codéthyline importantes sans morphine libre détectable, car leur taux de transformation en morphine est très faible $(22,25)$. Il faut donc :

- rechercher tous les opiacés et leurs métabolites, et doser tous ceux qui ont été détectés

- doser la morphine libre

- rechercher et doser la 6-monoacétylmorphine.

On peut donc raisonnablement proposer un seuil analytique pour la morphine de $5 \mathrm{ng} / \mathrm{mL}$ (tableau II).

\section{Cocaïne et ses métabolites}

La cocaïne est un stupéfiant illicite et en France aucun médicament n'en contient. L'administration se fait surtout par voie intra-nasale pour le chlorhydrate ou le sulfate de cocaïne, et par inhalation des vapeurs de cocaïne base pour le crack. La cocaïne a une demi-vie de 30 à 90 min (22) et ses métabolites principaux sont la benzoylecgonine (BZE) dont la demi-vie est de 5 à $7 \mathrm{~h}$, et l'ecgonine méthyl ester (EME) d'une demi-vie proche de $4 \mathrm{~h}$. Les proportions des deux métabolites dans le sang sont fonction du mode d'administration, sauf pour de fortes doses. La cocaïne est très instable in vitro et

Tableau II : Comparaison des seuils de positivité pour les stupéfiants dans le sang.

\begin{tabular}{|c|c|c|c|c|c|}
\hline \multirow[b]{2}{*}{ Famille de stupéfiants } & \multirow[b]{2}{*}{ Nom du composé selon la fiche $F$} & \multirow[b]{2}{*}{ Composés recherchés } & \multicolumn{3}{|c|}{ Seuil $(\mathrm{ng} / \mathrm{mL})$} \\
\hline & & & légal actuel & LDQ & proposé \\
\hline Cannabinoïdes & $\Delta-9-\mathrm{THC}$ & $\begin{array}{l}\Delta-9-\mathrm{THC} \\
\Delta-9-\mathrm{THC}-\mathrm{COOH} \\
11-\mathrm{OH}-\Delta-9-\mathrm{THC}\end{array}$ & 1 & 0,2 & $\mathbf{0 , 5}$ \\
\hline Cocaïne & cocaïne & BZE & 50 & 10 & 10 \\
\hline Amphétamines & amphétamine & $\begin{array}{l}\text { MDMA, MDA } \\
\text { Amphétamine }\end{array}$ & 50 & $5-20$ & 5 \\
\hline Opiacés & opiacés & $\begin{array}{l}\text { morphine } \\
\text { 6-MAM }\end{array}$ & 20 & 5 & 5 \\
\hline
\end{tabular}

LDQ : limite de quantification. Les autres abréviations sont données dans le texte. 
on ne la retrouve que très rarement dans le sang. En revanche on retrouve toujours la BZE et souvent l'EME. Une dizaine de métabolites mineurs peuvent être mis en évidence, dont l'ecgonine, l'ecgonidine et la norcocaïne. La méthylecgonidine (anhydroecgonine) signe la consommation de crack, car c'est un produit de la pyrolyse de la cocaïne: S'il y a association avec une boisson alcoolique, on pourra mettre en évidence le cocaéthylène, vraisemblablement toxique, formé par transméthylation hépatique avec l'éthanol $(22,26)$. On retrouve la BZE dans le plasma pendant les $24 \mathrm{~h}$ qui suivent la dernière consommation. Comme pour les cannabinoïdes, la concentration sanguine de BZE est mal corrélée aux effets psychotropes. Là aussi il y a un seuil analytique, car des concentrations de $10 \mathrm{ng} / \mathrm{mL}$ sont encore détectables. Les seuils sont comparés dans le tableau II.

\section{Conclusion}

De 1996 à 2003, les toxicologues analystes ont fait des progrès importants et ils savent maintenant doser les stupéfiants (cannabinoïdes, opiacés, métabolites de la cocaïne et amphétamines) dans le sang à des concentrations très basses. La participation active au programme de contrôle externe de qualité pour le dosage de stupéfiants dans le sérum, organisé annuellement par la Société Française de Toxicologie Analytique, y a très certainement contribué. Il s'avère que ces performances analytiques sont dépendantes de l'appareillage. Les textes réglementaires ont défini des seuils minima de détection et ne demandent pas d'interprétation des concentrations retrouvées. Il existe cependant des différences importantes entre ce qui est indiqué dans la fiche $\mathrm{F}$ et les seuils analytiques que nous proposons. Il était cohérent et raisonnable d'utiliser les seuils d'efficacité pharmacologique pour les substances dont on connaît les relations dose-effet (MDMA, morphine) et de prendre les seuils de quantification dans les cas où il n'existe pas de relation, ou si cette relation est variable selon les individus et le mode d'administration (cannabinoïdes, cocaïne). La dernière circulaire d'application a prévu de prendre en considération la «présence de stupéfiants dans le sang», ce qui permet de n'utiliser que des seuils analytiques, comme c'est le cas en Allemagne, en Belgique et en Suède. Ultérieurement, l'évolution des techniques et des connaissances permettra de les abaisser.

\section{Références}

1. Mura P., Papet Y., Pépin G., Deveaux M., Marquet P. Prévalence de la consommation de psychotropes illicites par les conducteurs en France et à l'étranger. Aspects réglementaires et propositions de la SFTA. Toxicorama $1996 ; 8(2): 5-10$.

2. Gaillard Y., Pépin G., Marquet P., Kintz P., Deveaux M., Mura P. Identification et dosage de la benzoylecgonine, cocaine, méthylecgonine-ester, codéine, morphine et 6MAM dans le sang total. Toxicorama $1996 ; 8(2): 17-22$.

3. Marquet P., Lachatre G., Kintz P., Pépin G., Deveaux M., Mura P. Identification et dosage des principales drogues amphétaminiques dans le sang total par CPG-SM. Toxicorama $1996 ; 8(2): 23-8$.

4. Kintz P., Cirimele V., Pépin G., Marquet P., Deveaux M., Mura P. Identification et dosage des cannabinoïdes dans le sang total. Toxicorama $1996 ; 8(2): 29-33$.

5. Deveaux M., HédouinV., Marquet P., Kintz P., Mura P., Pépin G. Conduite automobile et stupéfiants : procédures de dépistage et de dosage, accréditation des laboratoires. Toxicorama $1996 ; 8(2): 11-5$.

6. Décret $n^{\circ} 2001-751$ du 27 août 2001 relatif à la recherche de stupéfiants pratiquée sur les conducteurs impliqués dans un accident mortel de la circulation. Journal Officiel du 28 août 2001 ; 13759-61.

7. Arrêtés des 4 et 5 septembre 2001 fixant les modalités du dépistage des stupéfiants et des analyses et examens prévus par le décret $n^{\circ} 2001-751$ du 27 août 2001.

8. Loi $n^{\circ} 2003-87$ du 3 février 2003 relative à la conduite sous l'influence de substances ou plantes classés comme stupéfiants. Journal Officiel du 4 février 2003 ; 2103-4.

9. Décret $n^{\circ} 2003-293$ du 31 mars 2003 relatif à la sécurité routière. Journal Officiel du 1 avril $2003 ; 77: 5702$.

10. Circulaire d'application $n^{\circ}$ CRIM-2003.E8, publiée au Bulletin Officiel.

11. Loi n²001-1062 du 15 novembre 2001 relative à la sécurité quotidienne. Journal Officiel du 16 novembre 2001 ; 18215.

12. Deveaux M. L'alcool. In : Mura P. (coordinateur) Alcool, médicaments, stupéfiants et conduite automobile. Paris : Elsevier Option Bio, 1999 ; 3-19.

13. Verstraete A., Charlier C. Stupéfiants et conduite automobile : les actions réalisées en Belgique. Ann. Toxicol. Anal. $2003 ; 15(2): 128-37$.

14. Verstraete A. Fenêtres de détection des xénobiotiques dans le sang et les urines, la salive et la sueur. Ann. Toxicol. Anal. $2002 ; 14(4)$ : 390-4.

15. Mura P., Brunet B., Monzon C., Papet Y., Mauco G. Interprétation des concentrations sanguines en cannabinoïdes : données actuelles. Ann. Toxicol. Anal. 2003 ; 15(1) : 35-9.

16. Huestis M.A. Cannabis - Effects on human behavior and performance. Forensic Sci. Rev. 2002 ; 14 : 15-59.

17. Expertise collective INSERM. Cannabis. Quels effets sur le consommateur et la santé? Paris : Editions Inserm, 2001 ; 143-63. 
18. Pépin G., Mura P., Kintz P. et coll. Recherche de stupéfiants dans le sang de conducteurs d'automobiles : résultats d'une compilation française d'expertises toxicologiques. Toxicorama $1999 ; 11(1)$ : 12-6.

19. Mura P., Pépin G., Marquet P. Goullé J.P., Deveaux M., Tourneau J. Ghysel M.H., Molinaro R., Lhermitte M., Dumestre-Toulet V., Kintz P. Place des stupéfiants dans les accidents mortels et corporels de la voie publique. Résultats de 169 analyses sanguines réalisées en 1998 et 1999 à la demande d'une autorité judiciaire. Toxicorama $1999 ; 11(4): 225-31$.

20. Deveaux M., Gosset D. Les psychotropes haute couture. Ann. Toxicol. Anal. $2000 ; 12(1)$ : 90-6.

21. Ghysel M.H. Amphétamines et dérivés. In : Toxicologie et pharmacologie médicolégales. Kintz P. (coordinateur) Paris : Elsevier Option/Bio, 1998 ; 465-541.

22. Karch S.B. Karch's pathology of drug abuse, 3rd edition. Boca Raton : CRC Press, 2002 ; 541 pp.

23. Donnadieu S. Douleur du cancer : maniement des morphiniques forts . Concours Med. 2003 ; 125(18) : 881-5

24. Eichelbaum M, Evert B. Influence of pharmacogenetics on drug disposition and response. Clin. Exp. Pharmacol. Physiol. 1996 ; 23 : 983-5.

25. Pépin G. Opiacés et opioïdes. In : Toxicologie et pharmacologie médicolégales. Kintz P. (coordinateur) Paris : Elsevier Option/Bio, 1998 ; 335-430.

26. Ragoucy-Sengler C. Cocaïne. In : Toxicologie et pharmacologie médicolégales. Kintz P. (coordinateur) Paris : Elsevier Option/Bio, 1998 ; 431-64. 
Annexe : Fiches D, E et $F$ en vigueur en mai 2003.

\section{PROCÉDURE}

NOM de l'officier ou de l'agent de police judiciaire :

L_

Prênoms L

$N^{\circ}$ de la procédure $L$

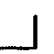

Signature :

\section{FICHE "D"*}

VÉRIFICATIONS CONCERNANT LES STUPÉFIANTS RÉSULTATS DES ÉPREUVES DE DÉPISTAGE

Références

Article L. 235-1 du Code de la Route

\section{PERSONNE CONCERNÉE}

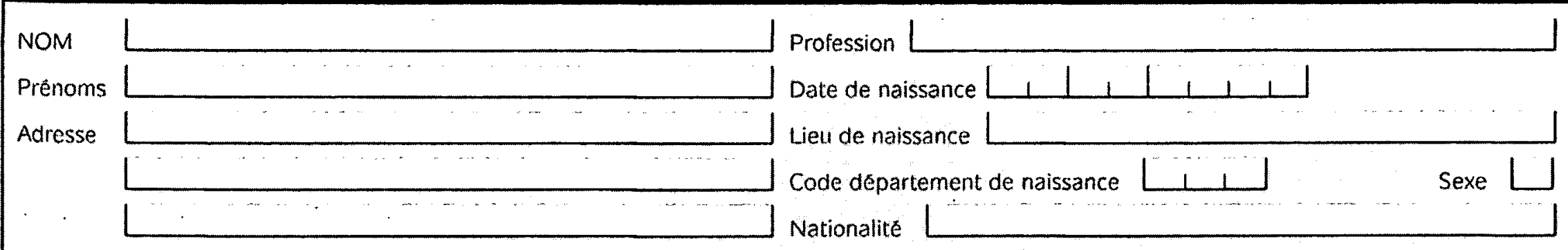

Code dẻpartement $\mid \begin{array}{llll}1 & 1 & 1\end{array}$

DÉPISTAGE DE STUPÉFIANTS

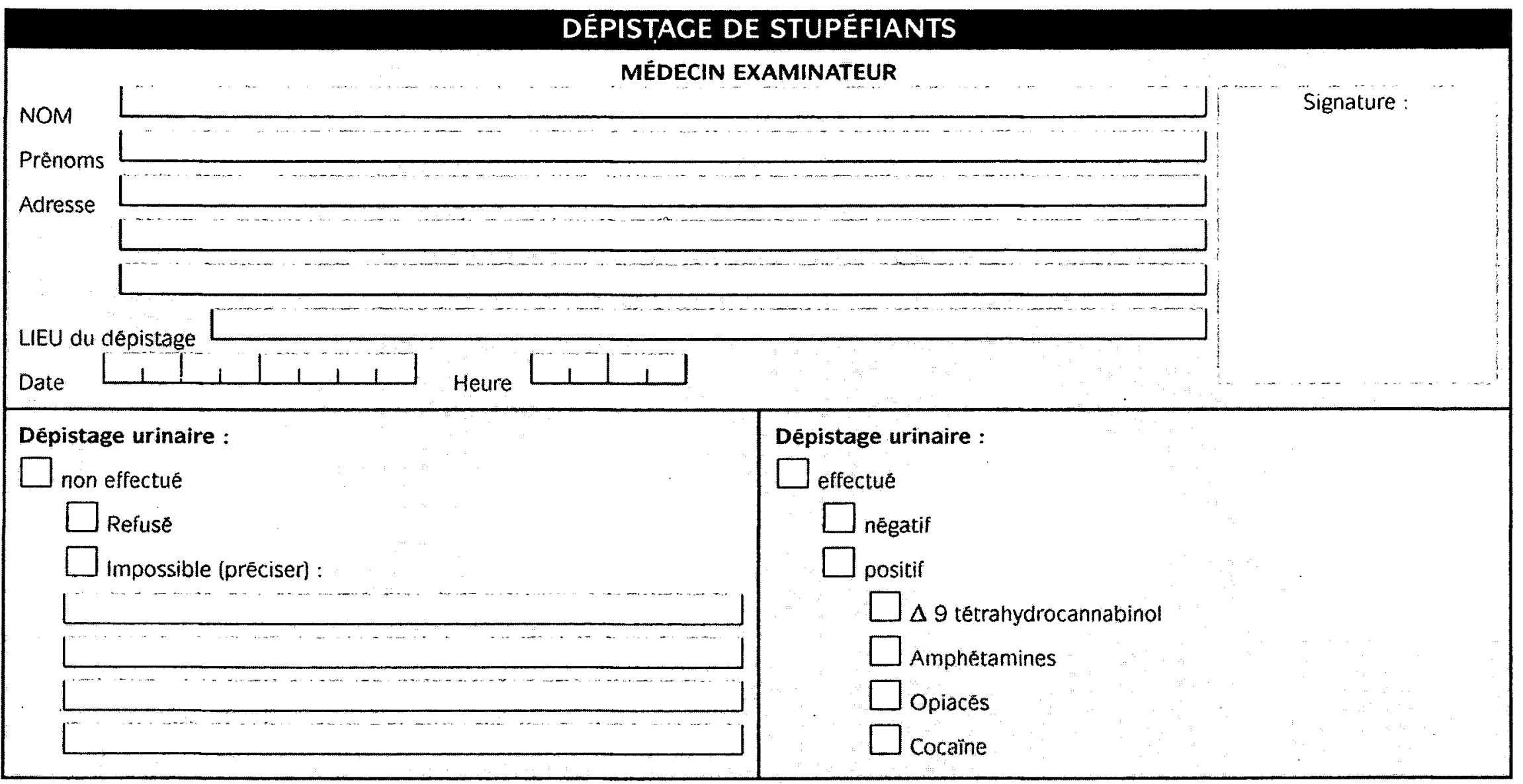

PRÉLĖVEMENT SANGUIN EN PRÉSENCE DE L'AUTORITÉ REQUÉRANTE

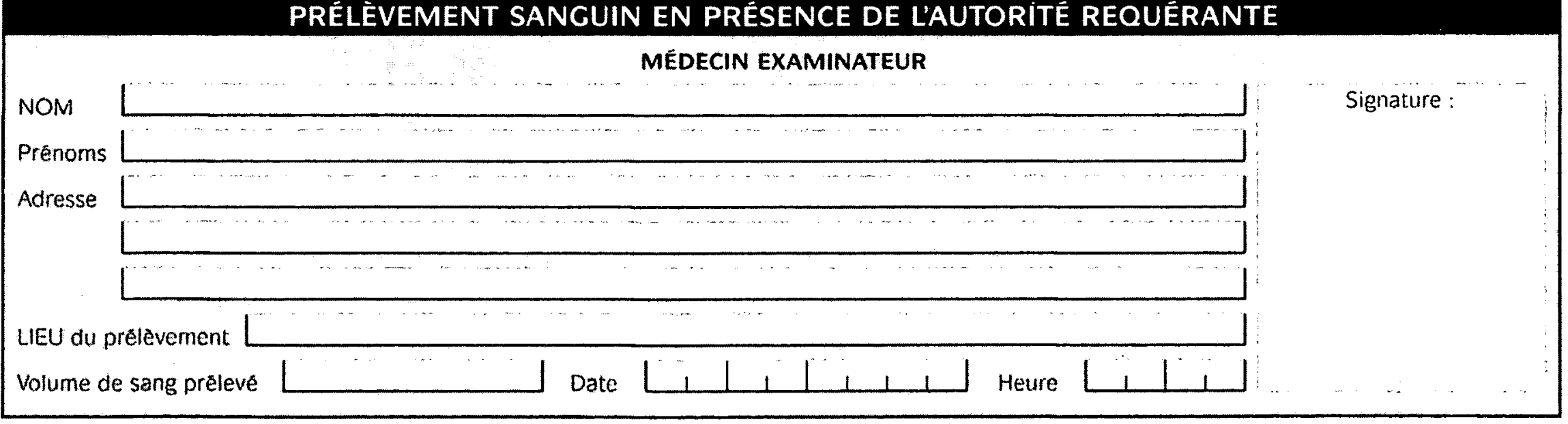

* Remplir cet imprimé au stylo bille pour une meilleure transcription.

remis au conducteur ayant subi les epreuves de depistage les $2^{\circ}$ et $3^{*}$ feuillets sont destinés à la procédure judiciaine le $4^{\circ}$ feuillet est destiné aux services de Police ou Gendarmerie et le $5^{\circ}$ feuillet à l'organisme d'etude. 


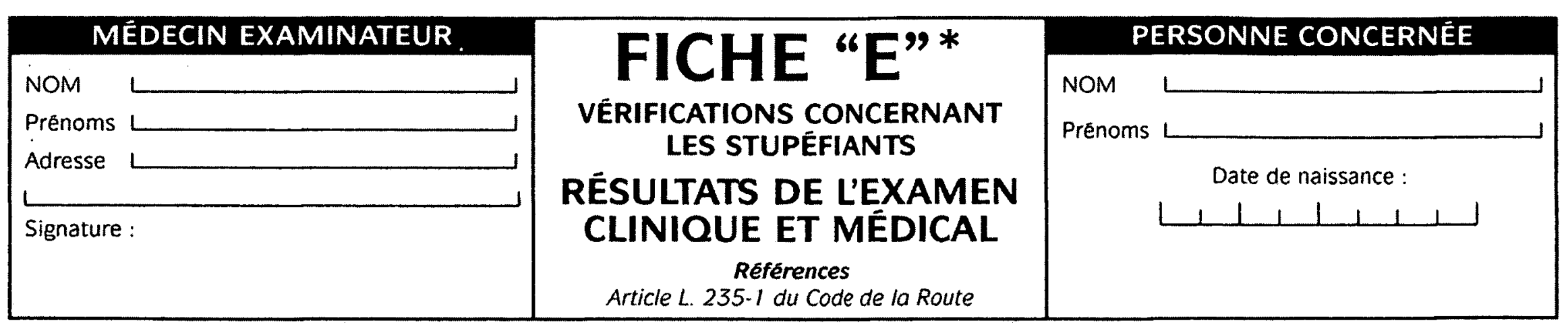

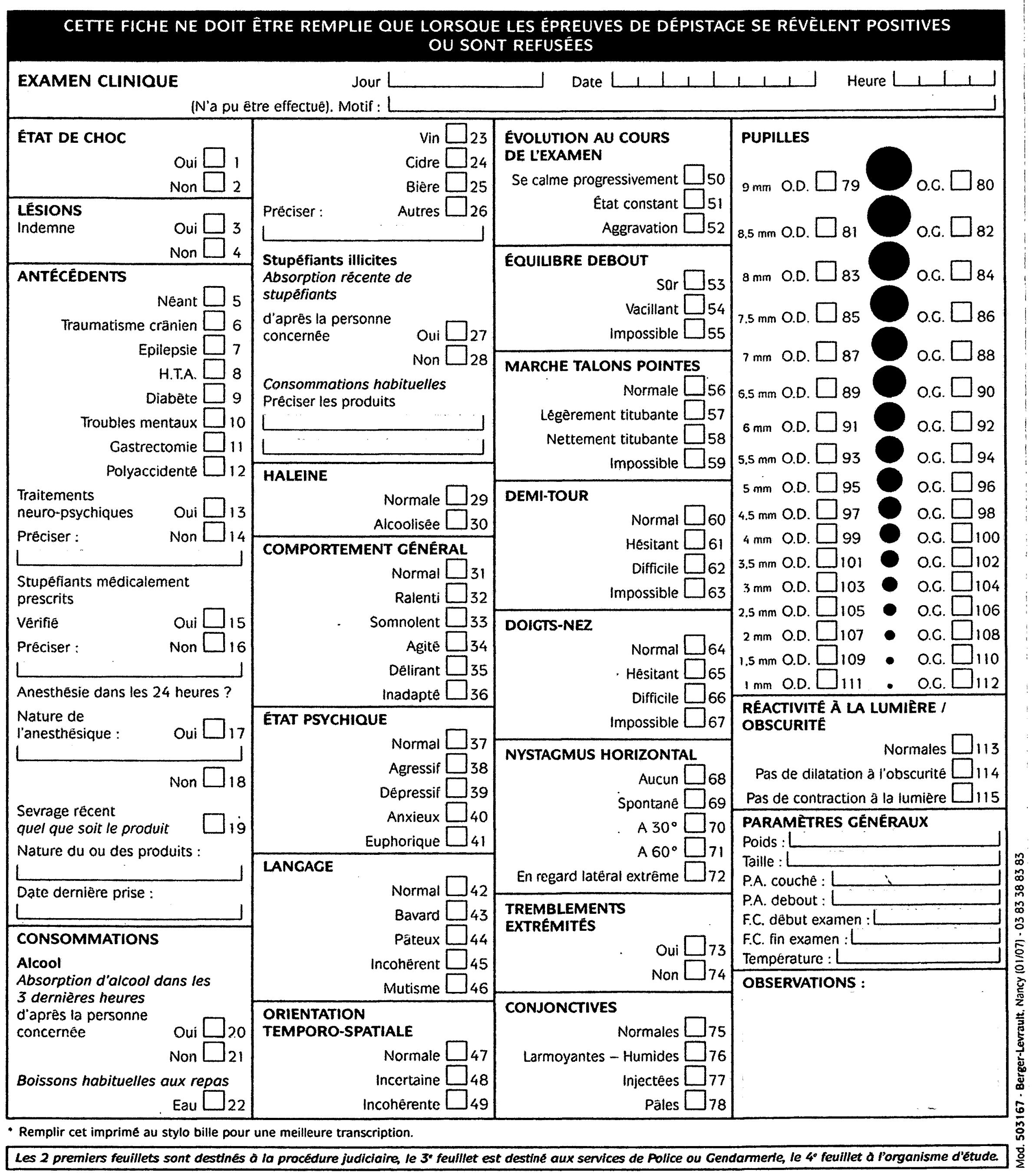




\section{PERSONNE CONCERNÉE}

Prénoms L

\section{FICHE “F”*}

\section{VÉRIFICATIONS CONCERNANT LES STUPÉFIANTS}

\section{RÉSULTATS DES ANALYSES DE SANG}

\section{Références}

Article L. 235-1 du Code de la Route

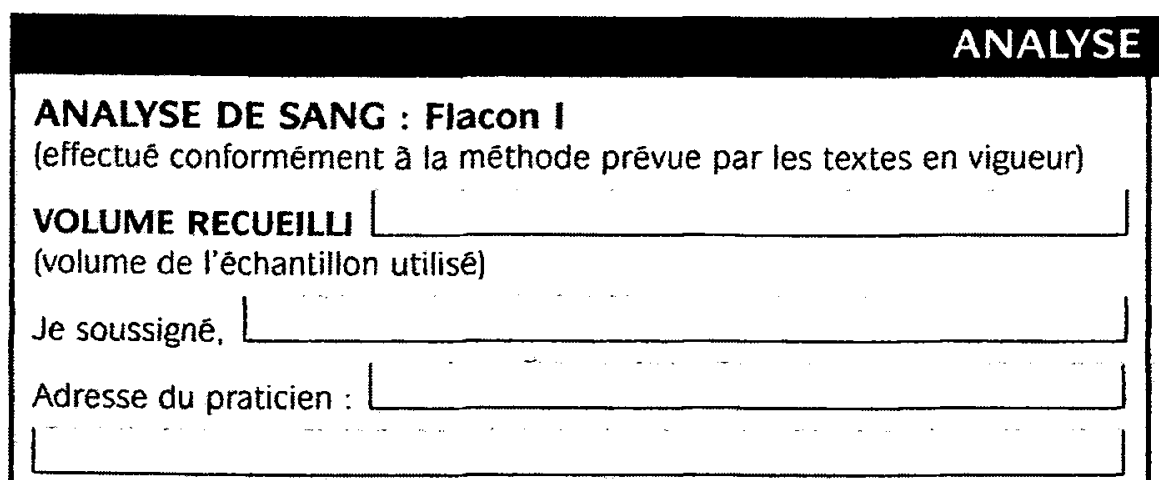

certifie avoir reçu l'ëchantillon le \begin{tabular}{llllllll}
\hline & & 1 & & 1 & 1 & 1 \\
\hline
\end{tabular}

à $L 111$ heures

État du scellé

\section{RECHERCHE ET DOSAGE DES STUPÉFIANTS}

Analyse: $\quad \square$ positive $\quad \square$ nēgative

Concentration :

$\square \Delta 9$ têtrahydrocannabinol

$\square$ Amphêtamine

$\square$ Opiäcēs

$\square$ cocaine

Observations :

L.

L

Signature et cachet du praticien :

\section{ANALYSE DE SANG : Flacon II (1)}

(effectué conformèment à la mêthode prévue par les textes en vigueur) VOLUME RECUEILLI L

(volume de l'êchantillon utilisé)

Je soussignè. L

Adresse du praticien :

L

certifie avoir reçu réchantillon le $\left[\begin{array}{lllllll}\hline & 1 & 1 & 1 & 1 & 1 & 1\end{array}\right]$

a $\lfloor+1.1\rfloor$ heures

État du scellê

RECHERCHE ET DOSAGE DES STUPÉFIANTS

Analyse: $\square$ positive . $\square$ négative

Concentration :

$\square \Delta 9$ tētrahydrocannabinol

$\square$ Amphêtamines

$\square$ Opiacés

$\square$ Cocainne

Observations :

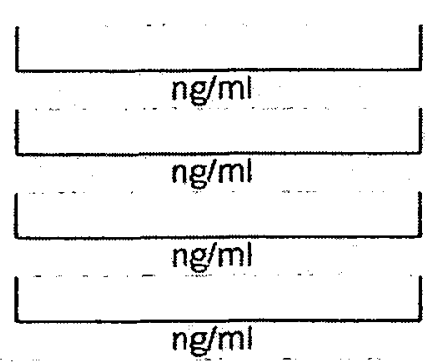

Signature et cachet du praticien :
RECHERCHE DES MÉDICAMENTS PSYCHOACTIFS en cas d'analyse de stupéfiants positive

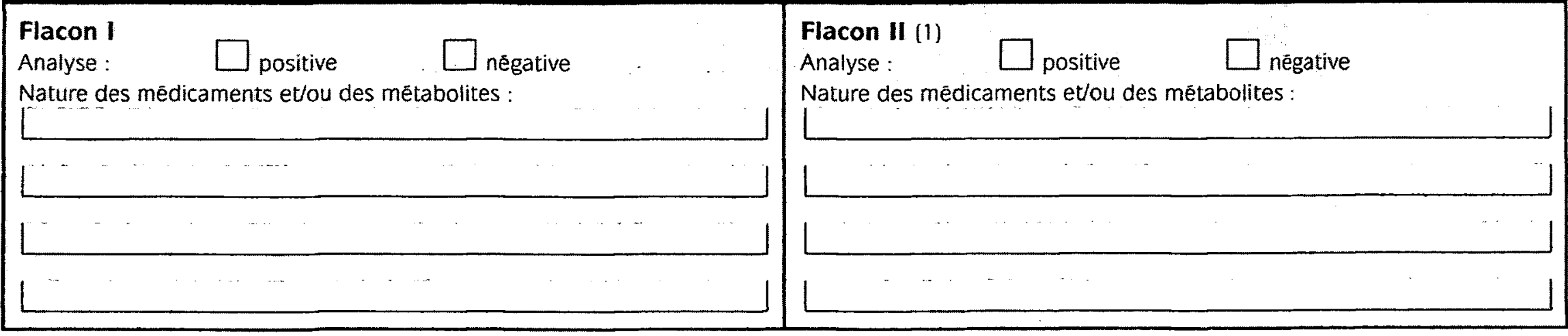

(1) En cas de demande d'une analyse de contrôle

* Remplir cet imprimé au stylo bille pour une meilleure transcription.

Les 2 premiers feuillets sont destinés à la procédure judiciaire, le 3' feuillet est destiné aux services de Police ou Gendarmerie, le $4^{*}$ feuillet à l'organisme d'étude 